\title{
Time-Varying Fine-Structure Constant Requires Cosmological Constant
}

\author{
Rainer W. Kühne \\ Vorm Holz 4, 42119 Wuppertal, Germany
}

Webb et al. presented preliminary evidence for a timevarying fine-structure constant. We show Teller's formula for this variation to be ruled out within the Einstein-de Sitter universe, however, it is compatible with cosmologies which require a large cosmological constant.

PACS numbers: 95.30.Sf, 95.30.Dr, 98.80.Es

The possibility of time-varying physical constants was suggested by Dirad 1 and Milne 3 . This suggestion has been widely discussed 10 for a number of motivations:

(i) The assumption of physical quantities being constant in space and time is ad hoc. It has to be either confirmed or rejected by observation 1 . 2.

(ii) The possibility of life as we know it depends on the values of a few basic physical constants and appears to be sensitive to their numerical valyes. This argument appeals to the anthropic principle 6 , 9 .

(iii) The numerical values of the basic dimensionless constants, e. g. the fine-structure constant, are not yet explained. If they depend on other constants and the age of the universe and are therefore time-varying, then the actual number of the independent free parameters of the standard theory of particle physics would be reduced.

Products and quotients of physical constants have been shown to be of fundamental importance for new physical phenomena:

(i) The unit of flux quantization in superconductors is $\phi=h /(2 e)$, where $h$ denotes the Planck constant and $e$ the unit electric charge 11,12. This finding was the first experimental proof of the famous BCS-theory 13 . Furthermore, the flux quantization in unitsof $\phi$ is of central importance for the Josephson junctions 14.

(ii) The characteristic magnetic flux in the AharonovBohm effect 15,16 is quantized in units of $\phi=h / e$.

(iii) The unit conductance in the integer quantum Hall effect 17 is $\hat{\sigma}=e^{2} / h$. In the fractional quantum Hall effect 18 the conductance is $\sigma=p e^{2} / h$, where $p$ is a rational number.

(iv) The Chandrasekhar limit mass 19 of white dwarf stars is

$$
M_{c}=\frac{3 \sqrt{\pi}}{2 \mu^{2} M_{p}^{2}}\left(\frac{\hbar c}{G}\right)^{3 / 2},
$$

where $\hbar=h /(2 \pi), c$ is the speed of light, $G$ is Newton's gravitational constant, $M_{p}$ is the proton rest mass, and $\mu$ is the number ratio of nucleons and electrons.

In order to find further fundamental and important physical phenomena one is-attempted to try to multiply or divide the Planck unit\$20,

$$
\begin{aligned}
l_{p} & =\left(G \hbar / c^{3}\right)^{1 / 2} \\
t_{p} & =\left(G \hbar / c^{5}\right)^{1 / 2} \\
m_{p} & =(\hbar c / G)^{1 / 2}
\end{aligned}
$$

with the Einstein constant 21 ,

$$
\kappa=8 \pi G / c^{4}
$$

the "natural units" $\hbar$ and $c$, and the present value $H_{0}$ of the Hubble parameter 22 . By just doing this, Teller 5 , 10 found the remarkable relation (in modern notation),

$$
\kappa m_{p} H_{0} c=\kappa \hbar H_{0} / l_{p}=8 \pi t_{p} H_{0}=\exp \left(-1 / \alpha_{0}\right),
$$

where

$$
\alpha_{0}=\frac{e^{2}}{4 \pi \varepsilon_{0} \hbar c}
$$

is the fine-structure constant. Here and in the following the subscript "0" refers to the present value of the respective parameter.

From Teller's equation one can derive the change rate

$$
\frac{\dot{\alpha}_{z}}{\alpha_{z}}=\alpha_{z} \frac{\dot{H}_{z}}{H_{z}},
$$

where the subscript " $z$ " denotes the value of the respective parameter at cosmological redshift $z$ and the dot denotes the derivative with time. This rate can be determined experimentally. By examining quasar absorption lines in the redshift region $0.5<z<1.6$, Webb et al.23 found preliminary evidence for a time-varying finestructure constant,

$$
\frac{\Delta \alpha}{\alpha}=(-1.09 \pm 0.36) \times 10^{-5}
$$

implying

$$
\frac{\dot{\alpha}_{z}}{\alpha_{z}}=-1 \times 10^{-5} H_{z} .
$$

If the preliminary finding by Webb et al. will not be confirmed by future investigations, then Eq. 10 should be considered as an upper limit for the change rate of the fine-structure constant.

It is our aim to examine which cosmologies are compatible with both Teller's equation and (the upper limit of) the change rate of the fine-structure constant determined by Webb et al.

Compatibility is satisfied for cosmologies like the de Sitter universe 24 and the steady state cosmology 25 , 
where the Hubble parameter is constant in time. Unfortunately, these models have already been ruled out as viable cosmologies, because they contradict a number of other cosmological finding, 27.

According to general agreement the expansion of the universe is explained by the Friedmann-Lemaitre equation 28 ,

$$
H^{2}=\frac{8 \pi G \varrho}{3}-\frac{k c^{2}}{R^{2}}+\frac{\lambda c^{2}}{3} .
$$

The curvature radius $R$ and the mean mass density $\varrho$ scale with the redshift as,

$$
\begin{gathered}
R=R_{0} /(1+z) \\
\varrho=\varrho_{0}(1+z)^{3} .
\end{gathered}
$$

By defining the present values of the mass parameter

$$
\Omega_{0}=\frac{8 \pi G \varrho_{0}}{3 H_{0}^{2}}
$$

and the cosmological parameter

$$
\lambda_{0}=\frac{\lambda c^{2}}{3 H_{0}^{2}}
$$

the Friedmann-Lemaitre equation can be written as 31 ,

$$
\left(\frac{H_{z}}{H_{0}}\right)^{2}=\Omega_{0}(1+z)^{3}-\left(\Omega_{0}+\lambda_{0}-1\right)(1+z)^{2}+\lambda_{0}
$$

The Einstein-de Sitter universe 32 , where $\Omega_{0}=1$ and $\lambda_{0}=0$, is a special case of the Friedmann-Lemaitre universe. In this cosmology, Eq. 16 is reduced to

$$
\left(\frac{H_{z}}{H_{0}}\right)^{2}=(1+z)^{3} \text {. }
$$

By using this equation Dysont 10 derived from Teller's equation the formula

$$
\alpha_{z}=\frac{\alpha_{0}}{1-\frac{3}{2} \alpha_{0} \ln (1+z)}
$$

which somewhat resembles expressions known from running coupling constants in quantum field theory. Dyson's equation (18) yields the rate

$$
\frac{\dot{\alpha}_{z}}{\alpha_{z}}=-\frac{3}{2} \alpha_{z} H_{z}
$$

and is, unfortunately, ruled out by the experimental result of Webb et al.23

By regarding Eq. 16 we find Teller's equation to be compatible with the observation by Webb et al. if the present value $\Omega_{0}$ of the mass parameter is extremely small and the cosmological parameter $\lambda_{0}$ slightly above unity.
The lower bound on $\Omega_{0}$ is given by the baryonic matter content of the universe. The visible baryonic matter of the universe was determined to be 33

$$
\Omega_{0}=0.003 h^{-1},
$$

where $h=H_{0} /(100 \mathrm{~km} /(\mathrm{s} \mathrm{Mpc}))$. (The parameter $h$ was introduced into cosmology for ease of notation; it should not be confused with the Planck constant.) This extremely small value of $\Omega_{0}$ is compatible with other determinations of $\Omega_{0}$ derived from examinations of the dynamics of clusters of galaxies if majority of their mass consists of a network of cosmic strings. This hypothetical network contributes to the local mass but do not influence the expansion of the universe 34 . Consequently, the mass contribution of the cosmic string network does not appear in the Friedmann-Lemaitre equation. Furthermore, determinations of $\Omega_{0}$ from hot big bang nucleosynthesis are model-dependent (especially the degree of inhomogeneities in the early universe is not well understood), for a discussion see Refs. 35 and 36.

If the observation by Webb et al. will be confirmed and Teller's equation is correct, then we can predict the following present values of cosmological parameters:

$$
\begin{aligned}
H_{0} & =69.7 \mathrm{~km} /(\mathrm{s} \mathrm{Mpc}) \\
\Omega_{0} & =0.004 \\
\lambda_{0} & =1.004
\end{aligned}
$$

This value of $H_{0}$ is compatible with majority of its recent determinations, e. g. Pierce et al 37 found $H_{0}=(87 \pm 7) \mathrm{km} /(\mathrm{s} \mathrm{Mpc})$, Freedman et al.38 reported $H_{0}=(80 \pm 17) \mathrm{km} /(\mathrm{s} \mathrm{Mpc})$, and Tanvir et al 39 measured $H_{0}=(69 \pm 8) \mathrm{km} /(\mathrm{s} \mathrm{Mpc})$. Harris et al 40 found $H_{0}=(77 \pm 8) \mathrm{km} /(\mathrm{s} \mathrm{Mpc})$ and Madore et al.41 reported $H_{0}=(73 \pm 21) \mathrm{km} /(\mathrm{s} \mathrm{Mpc})$.

Interestingly, the gesmological parameters of the BonnPotsdam model 31.4243 resemble those suggested above. Recently, van de Bruck 44.45 has examined the effects of the hypothetical cosmic string network on the cosmological velocity field within the framework of this model and has shown them to be compatible with cosmological observations.

The possible time-variation of the fine-structure constant implies the variation of at least one of the constants $c, \hbar, \varepsilon_{0}$, and $e$. A time-varying speed of light, for example, would imply a change in the radiation losses of accelerated charges, which would be of importance especially in the very early universe 46 .

In practice, time-variations of quantities with nonzero dimension can be measured and defined only relative to other quantities which have the same dimension. We will suggest a convention where the most fundamental constants are defined as being constant in time and can serve as a reference for the possible time-variation of other quantities.

We suggest to consider the "natural units" $c, \hbar, k_{B}$, and $\varepsilon_{0}$ as fundamental and constant in time. This is 
because according to relativity, lengths are measured as time intervals multiplied with the speed of light. Even our unit length, the meter, is defined as the length which the speed of light covers in 1/299 792458 seconds. Furthermore, quantum phenomena are described either by the particle picture or the wave picture, respectively. The "conversion factor" of some of the quantities of this duality is $\hbar$. Finally, temperature is a macroscopic quantity and can be defined by the energy of microscopic objects divided by the Boltzmann constant $k_{B}$.

According to concepts of quantum gravity, the Planck units, especially the Planck time, are most fundamental. Hence, we suggest to measure all quantities in units of the Planck units (and multiples thereof), $t_{p}, l_{p}=c t_{p}$, $m_{p}=\hbar /\left(c l_{p}\right), T_{p}=m_{p} c^{2} / k_{B}$, and $I_{p}=\left(\varepsilon_{0} \hbar c\right)^{1 / 2} / t_{p}$. Under these conventions, any time-variation of the finestructure constant implies the variation of the unit electric charge $e$.

${ }^{1}$ P. A. M. Dirac, Nature 139, 323 (1937).

${ }^{2}$ P. A. M. Dirac, Proc. R. Soc. A 165, 199 (1938).

${ }^{3}$ E. A. Milne, Proc. R. Soc. A 158, 324 (1937).

${ }^{4}$ P. Jordan, Die Herkunft der Sterne (Stuttgart, Vieweg, 1947).

${ }^{5}$ E. Teller, Phys. Rev. 73, 801 (1948).

${ }^{6}$ R. H. Dicke, Nature 192, 440 (1961).

${ }^{7}$ F. J. Dyson, Aspects of Quantum Theory, eds. A. Salam and E. P. Wigner (Cambridge, Univ. Press, 1972), p. 213.

${ }^{8}$ B. Carter, Confrontation of Cosmological Theories with Observation, ed. M. S. Longair (Dordrecht, Reidel, 1974), p. 291.

${ }^{9}$ B. J. Carr and M. J. Rees, Nature 278, 605 (1979).

${ }^{10}$ A. V. Ivanchik, A. Y. Potekhin, and D. A. Varshalovich, Astron. Astrophys. 343, 439 (1999).

${ }^{11}$ B. S. Deaver and W. M. Fairbank, Phys. Rev. Lett. 7, 43 (1961).

12 R. Doll and M. Näbauer, Phys. Rev. Lett. 7, 51 (1961).

13 J. Bardeen, L. N. Cooper, and J. R. Schrieffer, Phys. Rev. 108, 1175 (1957).

${ }^{14}$ B. D. Josephson, Phys. Lett. 1, 251 (1962).

${ }^{15}$ Y. Aharonov and D. Bohm, Phys. Rev. 115, 485 (1959).

${ }^{16}$ R. G. Chambers, Phys. Rev. Lett. 5, 3 (1960).

${ }^{17}$ K. von Klitzing, G. Dorda, and M. Pepper, Phys. Rev. Lett. 45, 494 (1980).

18 D. C. Tsui, H. L. Störmer, and A. C. Gossard, Phys. Rev. Lett. 48, 1559 (1982).

${ }^{19}$ S. Chandrasekhar, Astrophys. J. 74, 81 (1931).

${ }^{20}$ M. Planck, S.-B. Preuss. Akad. Wiss. (1899), p. 440.

${ }^{21}$ A. Einstein, Ann. Phys. (Leipzig) 49, 769 (1916).

${ }^{22}$ E. P. Hubble, Proc. Natl. Acad. Sci. 15, 168 (1929).

23 J. K. Webb et al., Phys. Rev. Lett. 82, 884 (1999).

${ }^{24}$ W. de Sitter, Konin. Ned. Akad. Wetenschappen 19, 1217 (1917).

${ }^{25}$ H. Bondi and T. Gold, Mon. Not. R. Astron. Soc. 108, 252
(1948).

${ }^{26}$ F. Hoyle, Mon. Not. R. Astron. Soc. 108, 372 (1948).

${ }^{27}$ P. J. E. Peebles et al., Nature 352, 769 (1991).

${ }^{28}$ A. Friedmann, Z. Phys. 10, 377 (1922).

${ }^{29}$ A. Friedmann, Z. Phys. 21, 326 (1924).

${ }^{30}$ G. Lemaître, Ann. Soc. Sci. Brux. 47, 49 (1927).

31 J. Hoell, D.-E. Liebscher, and W. Priester, Astron. Nachr. 315, 89 (1994).

32 A. Einstein and W. de Sitter, Proc. Natl. Acad. Sci 18, 213 (1932).

33 M. Persic and P. Salucci, Mon. Not. R. Astron. Soc. 258, 14 (1992).

34 A. Vilenkin, Phys. Rept. 121, 263 (1985).

${ }^{35}$ N. Hata et al., Phys. Rev. Lett. 75, 3977 (1995).

${ }^{36}$ C. J. Copi, D. N. Schramm, and M. S. Turner, Phys. Rev. Lett. 75, 3981 (1995).

${ }^{37}$ M. J. Pierce et al., Nature 371, 385 (1994).

${ }^{38}$ W. L. Freedman et al., Nature 371, 757 (1994).

39 N. R. Tanvir et al., Nature 377, 27 (1995).

${ }^{40}$ W. E. Harris et al., Nature 395, 45 (1998).

${ }^{41}$ B. F. Madore et al., Nature 395, 47 (1998).

42 D.-E. Liebscher, W. Priester, and J. Hoell, Astron. Astrophys. 261, 377 (1992).

43 D.-E. Liebscher, W. Priester, and J. Hoell, Astron. Nachr. 313, 265 (1992).

${ }^{44}$ C. van de Bruck, Phys. Rev. D 57, 1306 (1998).

${ }^{45}$ C. van de Bruck, Phys. Rev. D 57, 4663 (1998).

${ }^{46}$ D. V. Ahluwalia, unpublished (1999). 